\title{
Detection of neutral metastable fragments from electron-impact on argon clusters
}

\author{
P. J. M. van der Burgt ${ }^{\mathrm{a})}$ and J. W. McConkey \\ Department of Physics, University of Windsor, Windsor, Ontario, Canada N9B 3P4
}

(Received 16 August 1994; accepted 28 February 1995)

\begin{abstract}
We have studied the production of neutral metastable fragments in electron collisions with neutral argon clusters. The fragments are detected using a time-of-flight technique. The time-of-flight spectra show that the metastable fragments appear in two velocity ranges. Kinetic energy distributions are obtained, showing that the faster fragments are ejected with energies from 0.2 to $1.5 \mathrm{eV}$ and that the slower fragments have energies less than $0.2 \mathrm{eV}$. It is argued that the fragmentation of the clusters involves the excitation and decay of excitons in the clusters.The faster fragments are produced by $n=2$ excitons, which localize on an excimer or an excited trimer within the cluster and upon dissociation cause the ejection of a metastable atom. The slower fragments are produced by $n=1$ excitons, which tend to localize on the periphery of the cluster, leading to the ejection of a metastable atom due to weak repulsive forces with neighboring atoms. Four different production mechanisms for neutral metastable fragments are observed. (C) 1995 American Institute of Physics.
\end{abstract}

\section{INTRODUCTION}

Many studies of noble gas clusters have focused on the formation of clusters of different sizes in a free jet expansion. In order to observe clusters of different size, the clusters are first ionized by either electron or photon impact, and the resulting charged-cluster spectrum is measured with a mass spectrometer. It is now widely accepted that ionized cluster spectra are largely influenced by the relaxation of a positive molecular ion within the cluster. The molecular ion is formed by localization of a positive hole created by the ionization of the cluster $\left(\right.$ Haberland $^{42}$ ). While the original model evoked a dimer ion core, recent research has demonstrated that the localization more likely occurs on a larger unit. ${ }^{20,37,38,43-46}$ The relaxation releases about $1 \mathrm{eV}$ of energy to the cluster, causing it to heat up and evaporate several monomers before a stable configuration is reached. ${ }^{1-3}$ This process has been called metastable decay as the evaporation of monomers has been observed to occur on $\mu$ s time scales. Ion abundance anomalies in mass spectra of cluster beams are therefore caused by both the initial neutral cluster sizes produced in the free jet expansion and the delayed evaporation of monomers following ionization of the cluster.

Contrary to the detection of metastable cluster ions there are few experimental studies of excited neutral clusters or of the evaporated monomers produced after excitation/ ionization and relaxation of the clusters. Gspann and Vollmar ${ }^{4}$ have studied metastable excitations of very large clusters of $\mathrm{He}$ and $\mathrm{Ne}$ atoms. Buchenau et al. ${ }^{5}$ have studied excitation of He clusters following electron impact, while

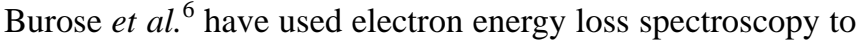
demonstrate the excitation of atoms on the surface and inside $\mathrm{Ar}$ and $\mathrm{Kr}$ clusters. Stapelfeldt et al. ${ }^{7}$ and Wörmer et al. ${ }^{8}$ have studied electronic excitation of rare gas clusters using fluorescence spectroscopy following absorption of synchro-

${ }^{a)}$ Present address: Department of Experimental Physics, St. Patrick's College, Maynooth, Co. Kildare, Ireland. tron radiation. Both Smith et al..$^{9}$ and Nagata and Kondow ${ }^{10}$ have measured kinetic energy distributions of neutral fragments produced by photodissociation of argon cluster ions.

While neutral monomers in the ground state can only be detected if they hit the detector with sufficiently high kinetic energy, metastable fragments are readily observed through electron emission from a metal surface. The purpose of the present study is to obtain information about the existence of neutral metastable fragments that result from fragmentation of excited and/or ionized clusters and thereby to acquire more insight into the fragmentation process.

Studies of neutral metastable fragments have been employed for some decades in the electron and photon impact dissociation of molecules. The abundant amount of information that has been obtained in these studies has been reviewed by Freund, ${ }^{11}$ Compton and Bardsley, ${ }^{12}$ and Zipf. ${ }^{13}$ The experimental technique involves the time-of-flight detection of neutral fragment atoms and molecules in metastable or high-lying Rydberg states. Fragments are flying towards the detector where detection proceeds through a mechanism of Auger de-excitation upon hitting the detection surface, thereby releasing an electron from the conduction band of the surface. The technique is therefore limited to states with comparatively long lifetimes $(t>1 \mu \mathrm{s})$ and with excitation energies $>8 \mathrm{eV}$ ( $5 \mathrm{eV}$ with specially prepared surfaces). With a simple transformation the time-of-flight distributions are transformed into kinetic energy distributions of the metastable fragments, which are obtained as a function of the electron impact energy. The information obtained is twofold. First, kinetic energy distributions can in some cases provide sufficient information to determine repulsive potential curves. Second, dissociation processes often produce onsets in the yield of metastables as a function of electron energy, and the dissociation processes involved can be deduced from the location of these onsets. In the dissociation process the fragments may pick up substantial kinetic energy. Kinetic energies from $0 \mathrm{eV}$ up to 15 or $20 \mathrm{eV}$ are observed in 


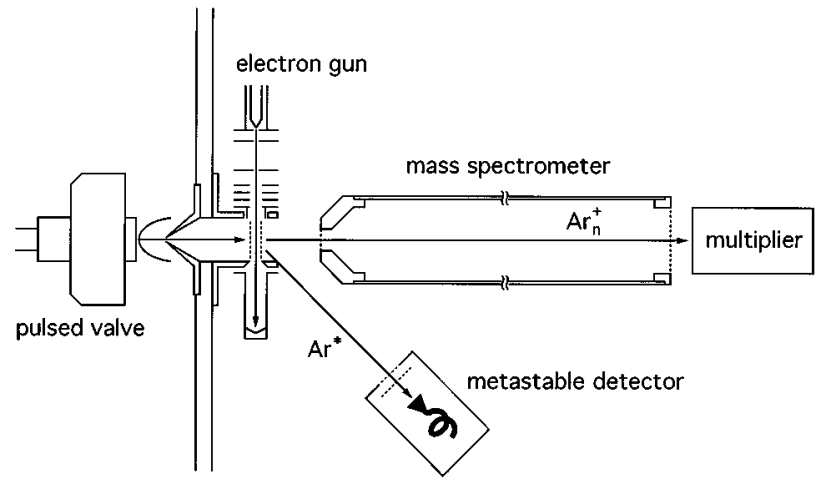

FIG. 1. Schematic diagram of the apparatus. The actual position of the electron beam is perpendicular to the plane containing the cluster beam and the metastable detector.

many molecular dissociations. The typical average kinetic energy of the fragments is of the order of a few eV.

In view of the success of neutral metastable fragment detection in the study of molecular dissociation it seems to be worthwhile to apply the same technique to the study of fragmentation of clusters. Argon clusters are very suitable for the initial study, because argon clusters are easily produced by supersonic expansion of argon gas, and the $\operatorname{Ar}^{*}\left(3 p^{5} 4 s\right)$ ${ }^{3} P_{0,2}$ states have suitable lifetimes (44.9 and 55.9 s) and excitation energies (11.72 and $11.55 \mathrm{eV})$ such as to be readily detected. The experiments on photodissociation of argon cluster ions by Smith et al. ${ }^{9}$ and Nagata and Kondow ${ }^{10}$ have demonstrated neutral photofragments with kinetic energies of up to $0.6 \mathrm{eV}$. In this paper we present the first measurements of kinetic energy distributions of neutral metastable fragments due to fragmentation of neutral argon clusters, which are of great interest in this context.

\section{EXPERIMENT}

The experimental setup is shown schematically in Fig. 1. A pulsed cluster beam is produced in the expansion chamber by expanding the gas into the vacuum through a pulsed valve with a $0.25 \mathrm{~mm}$ conical nozzle. All measurements presented in this paper have been performed with a stagnation pressure of $3.5 \mathrm{~atm}$ and a stagnation temperature of $295 \mathrm{~K}$ (room temperature). The piezoelectric valve is operated with a pulse width of $150 \mu$ s and a repetition rate of $40 \mathrm{~Hz}$. The repetition rate is limited by the pumping speed of the pumps on the expansion chamber. The nozzle is at a distance of 15 $\mathrm{mm}$ from a skimmer of $1 \mathrm{~mm}$ diam which separates the expansion chamber from the collision chamber. In the course of the study we found that the performance of the valve significantly depends on the amplitude of the pulse applied to it and also changes with time. In the measurements reported here we have adjusted the valve pulse so as to keep the (time-averaged) pressure in the expansion chamber at a constant value of $8.0 \times 10^{-5}$ Torr.

In the collision chamber, at a distance of $53 \mathrm{~mm}$ from the skimmer, the cluster beam is crossed at right angles with an electron beam. Cluster ions are detected using a time-offlight mass spectrometer ${ }^{14,17}$ which is mounted along the axis of the cluster beam. Neutral metastable fragments are de-
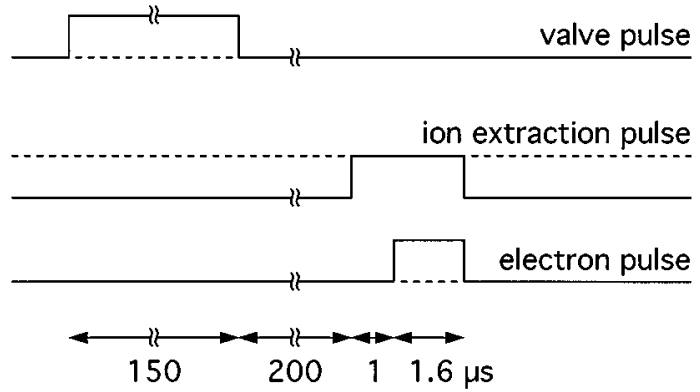

FIG. 2. Time sequence of the pulses applied to the different elements of Fig. 1. As discussed in the text, the pulse of electrons coincides with the center of the gas pulse as it traverses the interaction region.

tected by using a channeltron with appropriately biased meshes in front to prevent charged particles from being detected. The neutral metastable detector is located in a plane perpendicular to the electron beam and at either a $45^{\circ}$ angle or a $90^{\circ}$ angle with the cluster beam. Both the neutral metastable detector and the electron gun have been used in earlier studies of dissociation of molecules. ${ }^{15,16}$

Figure 2 shows the pulse sequence used. A $150 \mu$ s wide pulse is applied to the piezoelectric valve. Synchronously with the valve pulse the electron gun is pulsed with a $1.6 \mu \mathrm{s}$ wide pulse. The timing of this pulse is such that the electrons hit the clusters at about $80 \mu \mathrm{s}$ after the start of the cluster pulse. The electron pulse is chosen much narrower than the cluster pulse in order to obtain the necessary time resolution in the time-of-flight spectrometers.

Figure 3 (top) shows a mass spectrum obtained with a stagnation pressure of $3.5 \mathrm{~atm}$, indicating that smaller cluster sizes are abundantly produced by our source. We can estimate the average cluster size in the beam by using scaling laws developed by Hagena. ${ }^{18}$ The reduced scaling parameter $\Gamma^{*}$ is calculated using the equation [see Eq. (1) of Wörmer et al. ${ }^{19}$ ]

$$
\Gamma^{*}=k d_{\mathrm{eq}}^{0.85} \rho_{0} / T_{0}^{2.29},
$$

where $d_{\text {eq }}$ is the equivalent nozzle diameter in $\mathrm{mm}, \rho_{0}$ is the stagnation pressure in mbar, $T_{0}$ is the source temperature in $\mathrm{K}$, and $k=1646$ for argon. For the present experiment we find $\Gamma^{*}=1000$. The graph in Fig. 1 of Wörmer et al. ${ }^{19}$ indicates an average cluster size of about 100.

Because the mass spectrometer is mounted in line with the cluster beam, the velocity of neutral clusters and cluster fragments can be measured as well. This is done by setting the second grid at $0 \mathrm{~V}$ and the flight tube at $+5 \mathrm{~V}$ (to prevent detection of positive ions). Figure 3 (bottom) shows a timeof-flight spectrum of neutral excited fragments generated by impact of $50 \mathrm{eV}$ electrons. Fitting of a supersonic velocity distribution (see Haberland $e t$ al. ${ }^{51}$ ) shows that the mean velocity of the clusters is $602 \mathrm{~m} / \mathrm{s}$ with a FWHM of $85 \mathrm{~m} / \mathrm{s}$, the Mach number is 12.7 , and the expansion temperature is 6.3 $\mathrm{K}$. The velocity distribution obtained agrees well with cluster velocities found in other similar experiments. The velocity distribution has been measured several times in the course of this study (always at a stagnation pressure of $3.5 \mathrm{~atm}$ ). In all 

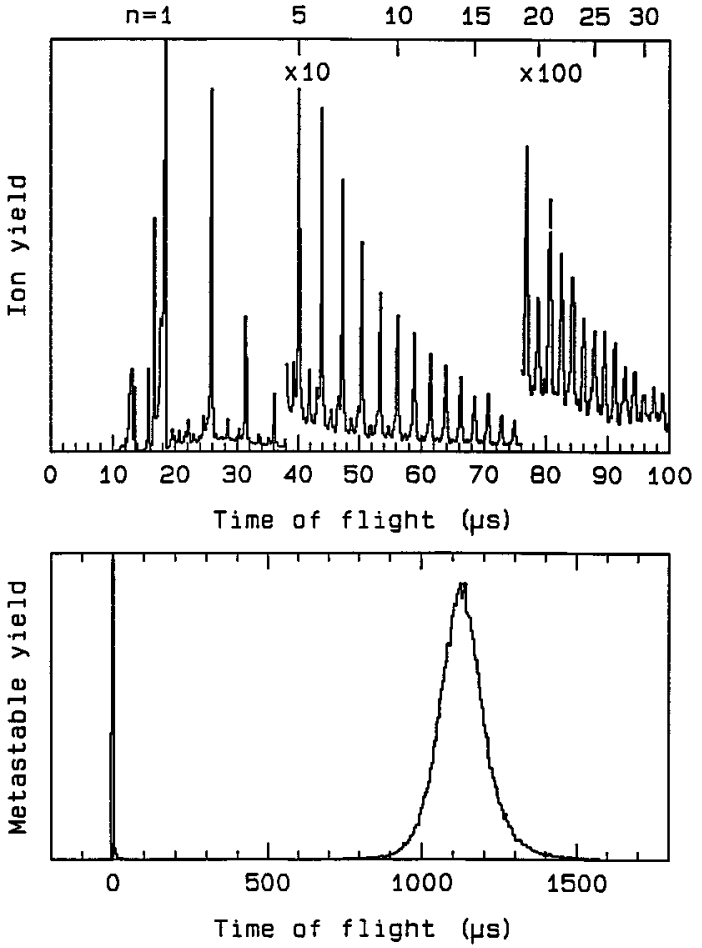

FIG. 3. (Top) Time-of-flight spectrum of ionized species obtained at $50 \mathrm{eV}$ electron impact and an Ar stagnation pressure of $3.5 \mathrm{~atm}$. The cluster sizes are indicated. (Bottom) Time-of-flight spectrum of neutral metastable fragments detected by the multiplier when the mass spectrometer flight tube is held at $+5 \mathrm{~V}$. The peak at zero time is due to photons resulting from the interaction of the electron beam pulse with the cluster beam.

cases the mean velocity obtained was very close to $600 \mathrm{~m} / \mathrm{s}$, and this value is used in the analysis of the measurements.

Except for the velocity measurement described above, all measurements on neutral metastable fragments have been performed using the neutral metastable detector (channeltron), which is located at a distance of $124 \mathrm{~mm}$ from the interaction region. During these measurements the voltages on the mass spectrometer were set to zero.

Excitation functions for neutral metastable fragments have been measured as well. Usually excitation functions are measured successively by detecting only those fragments that arrive at the detector within well defined time-of-flight windows. In the present experiment all excitation functions have been measured simultaneously by computer controlled operation of the multichannel scalar used for data accumulation. ${ }^{16}$

Calibration of the electron energy is obtained by running the pulsed valve with $3.5 \mathrm{~atm}$ of helium. No indication of clustering of helium has been observed. Two excitation functions are measured in this situation; one for the photons and one for the metastables. Comparisons of the observed thresholds with known values for the excitation energies of $\mathrm{He}\left(2{ }^{1} P\right)$ and $\mathrm{He}\left(2{ }^{3} S\right)$ provide the calibration.

\section{RESULTS}

\section{A. Scale transformations}

In interpreting the time-of-flight spectra of the metastable fragments we have to consider that the velocity $w$ with

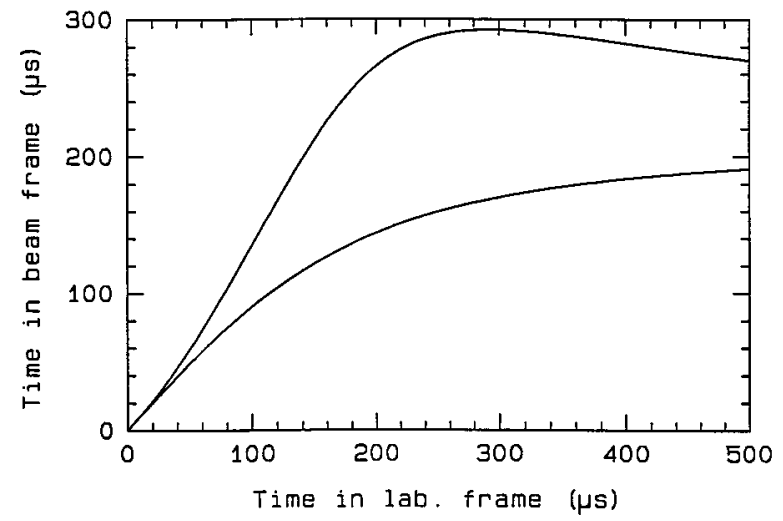

FIG. 4. Curves relating time of flight in the laboratory frame with time of flight in a frame of reference traveling with the cluster beam (see text). The top curve refers to the detector at $45^{\circ}$ and the bottom curve to the detector at $90^{\circ}$.

which metastable fragments reach the detector is the vector sum of the velocity of the parent clusters $v=600 \mathrm{~m} / \mathrm{s}$ and the velocity $u$ the fragment has acquired in the fragmentation process. We can correct for this effect by a scale transformation from the laboratory frame to the beam frame which is stationary with respect to the parent clusters. The transformation is carried out by using the following equation, relating the measured flight time $t_{M}=l / w$ to the flight time in the beam frame $t=l / u$ (the flight time the fragment would need to travel the same distance in the beam frame),

$$
t=t_{M}\left[1-2 \cos \theta v t_{M} / l+\left(v t_{M} / l\right)^{2}\right]^{-1 / 2},
$$

where $l$ is the distance to the detector and $\theta$ is the angle between the direction of the detector and the cluster beam. Figure 4 shows the relation between $t$ and $t_{M}$ for the two different detector angles $\theta=45^{\circ}$ and $90^{\circ}, l=124 \mathrm{~mm}$, and $v=600 \mathrm{~m} / \mathrm{s}$.

In applying such a transformation we make a tacit assumption. Consider two fragments with unequal velocities $w_{1}$ and $w_{2}$ in the laboratory frame. If both fragments are detected, $w_{1}$ and $w_{2}$ point in the same direction. A simple vector diagram shows that the corresponding velocities $u_{1}$ and $u_{2}$ in the beam frame point in different directions. It follows that the above transformation is only valid assuming that the angular distribution of the fragments in the beam frame (i.e., in reference to the target) is isotropic.

The kinetic energy of the fragments is obtained by a subsequent transformation from flight time $t$ in the beam frame to fragment kinetic energy $E$,

$$
E=1 / 2 m u^{2}=1 / 2 m l^{2} t^{-2} .
$$

Applying a very nonlinear transformation to a spectrum results in many data points and large scatter in one end of the transformed spectrum. To avoid this both transformations have been applied to the measured spectra by using equidistant points on the transformed scale and summing over (fractions of) channels in the original spectrum. 


\section{B. Time-of-flight spectra}

Time-of-flight spectra of neutral metastable fragments for different electron impact energies are shown in Figs. 5(a) and 5(b). The origin of the time-of-flight scale is determined by the prompt peak produced by radiative decay of excited clusters and fragments.

Two features are present in the series of spectra in Fig. 5. The first feature is narrow and has a maximum around $60 \mu \mathrm{s}$. The second feature is much broader and has a maximum that shifts from about $220 \mu \mathrm{s}$ (at $13 \mathrm{eV}$ ) to $160 \mu \mathrm{s}$ (at $200 \mathrm{eV}$ ).

Due to the very well directed nature of the cluster beam, no signal should be observed at the detector, positioned at $45^{\circ}$ or $90^{\circ}$ to the cluster beam direction, unless some additional directed motion occurs. The only possibilities of metastable fragments moving towards the detector are either because of excitation of randomly directed background gas atoms or because break-up of a cluster has occurred, imparting kinetic energy to a fragment and causing it to move out of the cluster beam. The first possibility has been demonstrated to be a minor one in the following way.

The time-of-flight spectra (Fig. 5) have been taken with a $350 \mu$ s delay between the valve pulse and the electron beam pulse, so that the electrons are hitting the clusters at a time midway in the cluster pulse. We have also taken a few spectra (not shown in Fig. 5) with a delay of $500 \mu$ s so that the gas pulse is effectively over. Metastable spectra taken this way show only a contribution of neutral metastable argon atoms produced by electron impact of argon atoms remaining in the chamber just after the cluster pulse has finished. The spectra taken this way show a Maxwellian distribution with a maximum near $260 \mu$ s, that remains in the same place when the electron energy is increased. Keeping this in mind we come to the following conclusions regarding the spectra in Fig. 5. The first feature, appearing at smaller flight times, must with certainty be due to fragmentation of clusters. The second feature may have a small contribution due to background argon atoms being excited, but because the maximum in the distribution shifts from 220 to $160 \mu \mathrm{s}$, here also the largest contribution must be contribution from argon clusters.

Careful comparison of Figs. 5(a) and 5(b) reveals several differences. The maximum in the first feature occurs at slightly lower flight times at $45^{\circ}$ compared to $90^{\circ}$, especially at lower electron impact energies. The second feature is clearly much less pronounced in the data from the $90^{\circ}$ detector, reflecting the fact that the cutoff in velocities of fragments which can reach the detector is much more severe at $90^{\circ}$.

We have applied the $t_{M} \rightarrow t$ transformation from laboratory time-of-flight to time-of-flight with respect to the cluster beam to the two TOF spectra taken at $29 \mathrm{eV}, 45^{\circ}$ and $31 \mathrm{eV}$, $90^{\circ}$. The transformation has been performed for a cluster beam velocity of $v=600 \mathrm{~m} / \mathrm{s}$, and the result is shown in Fig. 6. There is very good agreement in the shapes of the first feature, consistent with the assumption that this feature is from decay of parent states with a velocity of $600 \mathrm{~m} / \mathrm{s}$. When the same transformation is applied to TOF spectra at incident electron energies of $50 \mathrm{eV}$ and higher, the agreement is less good. This may very well be due to an angular anisotropy of
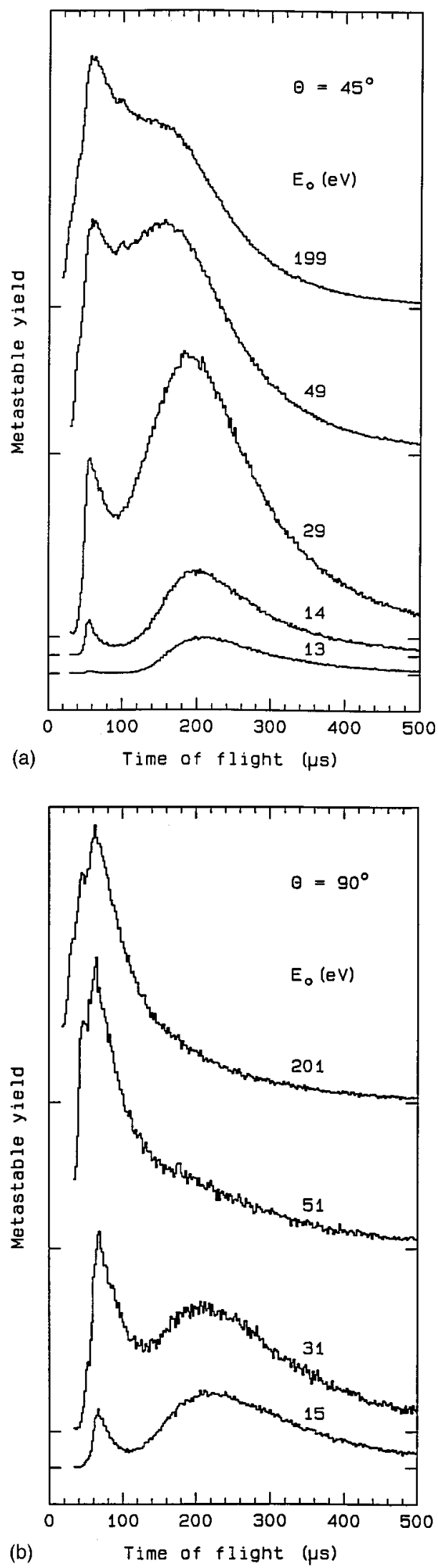

FIG. 5. (a) Time-of-flight spectra for neutral metastable fragments obtained with the detector positioned at $45^{\circ}$ with respect to the cluster beam. The incident electron beam energies are noted. (b) Similar, except with the metastable detector positioned at $90^{\circ}$. Horizontal scales indicate laboratory timeof-flight. 


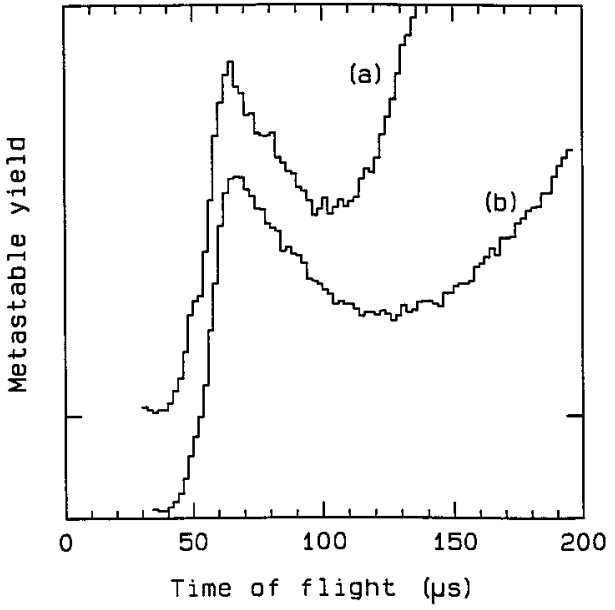

FIG. 6. Comparison of time-of-flight (a) obtained at an incident electron energy of $31 \mathrm{eV}$ with the metastable detector at $90^{\circ}$, and (b) at $29 \mathrm{eV}$ and $45^{\circ}$. The transformation from laboratory time-of-flight to beam time-offlight has been applied to both spectra (see text).

fragments produced by the additional fragmentation process occurring at electron impact energies above $30.8 \mathrm{eV}$ (process 2, see Sec. IV).

The divergence between the spectra in Fig. 6 at flight times above $120 \mu \mathrm{s}$ shows that the transformation does not work very well for the second feature. The relation between $t_{M}$ and $t$ at $45^{\circ}$ (top curve in Fig. 4) suggests that a continuous distribution of fragments near $t=290 \mu$ s would result in a distribution in the $45^{\circ}$ detector with a sharp dip at $t_{M}=292$ $\mu$ s. Such a dip is not observed in the time-of-flight spectra of Fig. 5, because it is effectively washed out by the spread in forward velocities of the parent clusters. The $t_{M} \rightarrow t$ transformation assumes that all clusters have a velocity of exactly $600 \mathrm{~m} / \mathrm{s}$ along the $x$-axis. It is therefore accurate for the faster fragments, but becomes less reliable for fragments ejected with velocities comparable to the beam velocity of $600 \mathrm{~m} / \mathrm{s}$.

Assuming that the incident electron only imparts excitation energy to the cluster and does not affect the subsequent relaxation and fragmentation, the TOF distribution of fragments from a single fragmentation process is expected to be independent of the incident electron energy. If we assume that at the lowest energies $(13-15 \mathrm{eV})$ the second feature is dominated by a single process, then this contribution may be subtracted from the time-of-flight spectra at higher energies.

It turns out that the second feature at $13-15 \mathrm{eV}$ is very well fitted by a Gaussian velocity distribution

$$
g(w)=A \exp \left[-4 \ln 2\left(\frac{w-w_{0}}{\Delta w}\right)^{2}\right],
$$

where $w_{0}$ and $\Delta w$ are the average velocity and the FWHM of the distribution (with respect to the laboratory frame).

We have fitted this function (transformed to a time-offlight scale) to the spectrum at $13 \mathrm{eV}, 45^{\circ}$. The same function has been fitted to each of the other time-of-flight spectra at $45^{\circ}$, such that $w_{0}$ and $\Delta w$ were fixed and only $A$ was adjusted to fit the spectrum between 300 and $500 \mu$ s. Subse-

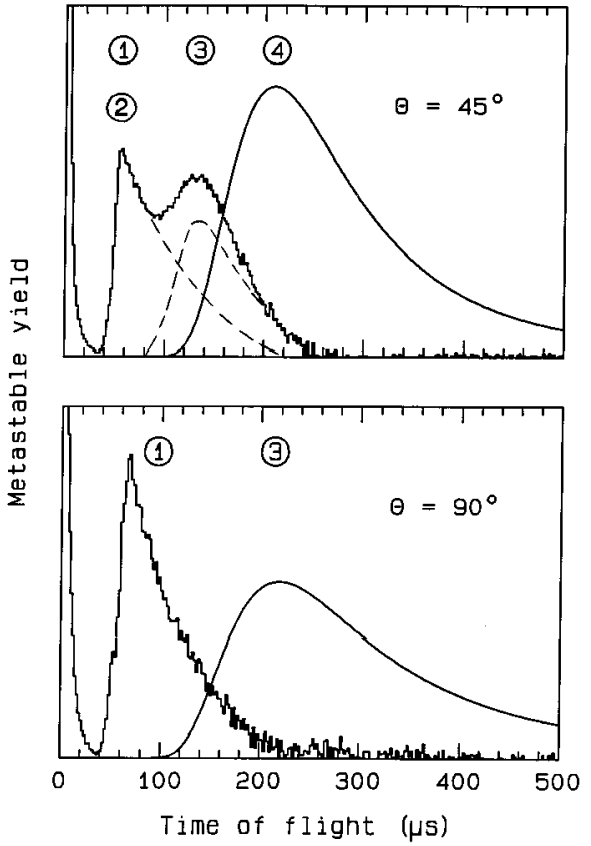

FIG. 7. (Top) Time-of-flight spectrum at $29 \mathrm{eV}, 45^{\circ}$ obtained after subtraction of the fit of the spectrum at $13 \mathrm{eV}, 45^{\circ}$. The fit is represented by the full curve. The dashed curves indicate a possible deconvolution of the contributions of the various processes (numbered 1-4) to the spectrum. (Process 2 has an onset at $30.8 \mathrm{eV}$ and only contributes to the feature at higher energies.) (Bottom) Time-of-flight spectrum at $31 \mathrm{eV}, 90^{\circ}$ obtained after subtraction of the fit of the spectrum at $15 \mathrm{eV}, 90^{\circ}$. Horizontal scales indicate laboratory time-of-flight.

quently the fits were subtracted. In a similar manner the fit to the spectrum at $15 \mathrm{eV}, 90^{\circ}$ has been subtracted from the other time-of-flight spectra at $90^{\circ}$.

Figure 7 compares the spectra at $29 \mathrm{eV}$, and $45^{\circ}$ and 31 $\mathrm{eV}, 90^{\circ}$, obtained after subtraction of the fits. The spectra clearly show that at $45^{\circ}$ two processes contribute to the second feature, whereas at $90^{\circ}$ only one process contributes. The first feature is produced by two processes also (see next section). Thus a total of four processes appear to be responsible and the corresponding time-of-flight structures are labeled $1-4$ in Fig. 7.

Process 4 is absent in the $90^{\circ}$ time-of-flight spectra which can be understood by looking at Fig. 4. Process 4 produces fragments with velocities less than $620 \mathrm{~m} / \mathrm{s}$ (less than $0.08 \mathrm{eV}$ kinetic energy). These fragments would have flight times with respect to the beam frame longer than 200 $\mu \mathrm{s}$, providing a significant contribution above $140 \mu \mathrm{s}$ in the time-of-flight spectra at $45^{\circ}$. However, the curves in Fig. 4 shows that these fragments cannot be observed at $90^{\circ}$.

Figure 7 shows that the fragments produced by process 3 have a significant difference in flight time between $45^{\circ}$ and $90^{\circ}$. This can be understood in a similar manner. Suppose the fragments produced by process 3 have kinetic energies between 0.29 and $0.035 \mathrm{eV}$, their flight times with respect to the beam frame would be between 105 and $300 \mu$ s. The curves in Fig. 4 show that they would appear between 80 and 500 $\mu$ s at $45^{\circ}$, and between 120 and $500 \mu$ s at $90^{\circ}$, in agreement with the observations in Fig. 7. 


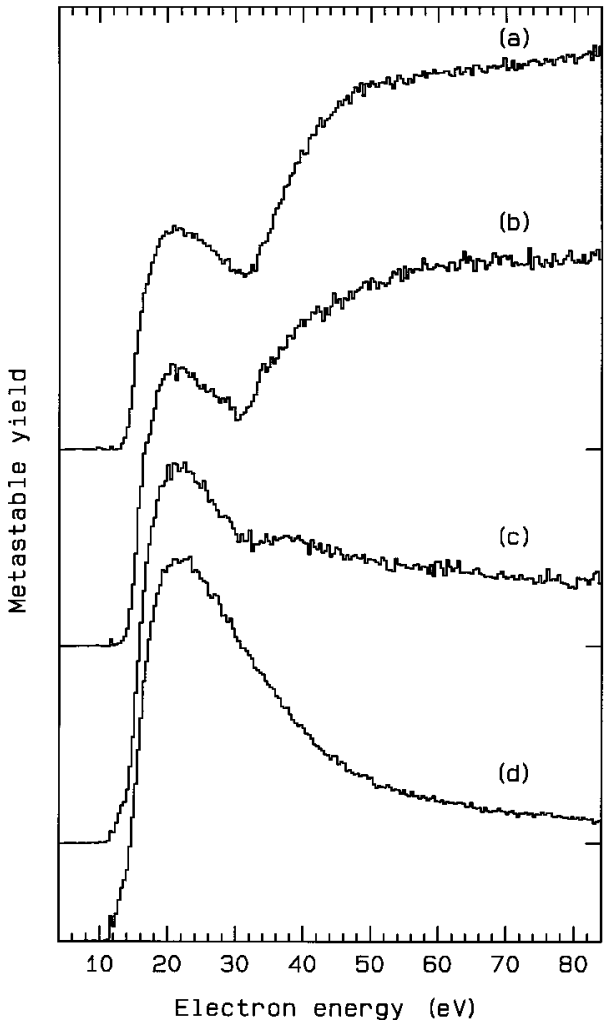

FIG. 8. Excitation functions with the metastable detector positioned at $90^{\circ}$, appropriate to the different time-of-flight regions of Fig. 5. (a) 40-90 $\mu \mathrm{s}$; (b)

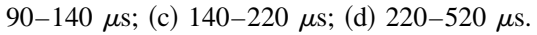

\section{Excitation functions}

We have measured a set of 50 excitation functions for 10 $\mu$ s wide time-of-flight windows with the metastable detector at $90^{\circ}$. The excitation functions in Fig. 8 have been generated by adding excitation functions for adjacent time-of-flight windows from this set.

The four excitation functions displayed in Fig. 8 are for four different time-of-flight regions chosen to separate the various processes shown in Fig. 7 as much as possible. The top function in Fig. 8(a) shows an excitation function dominated by two processes. Process 1 has an onset at $13.7 \mathrm{eV}$ and its excitation function rises sharply from threshold to a maximum at $20 \mathrm{eV}$. The second process has a threshold at $31.3 \mathrm{eV}$ and has a maximum at much higher energy (perhaps near $100 \mathrm{eV}$ ).

The bottom function in Fig. 8(d) is qualitatively similar to the excitation of argon atoms by electron impact. The observed onset is at $11.7 \mathrm{eV}$. Based on Fig. 7 it appears that this excitation function is dominated by process 3 . Data from 30 adjacent time-of-flight windows have been combined to obtain this excitation function. The excitation functions for the individual $10 \mu$ s windows show that the falloff with increasing energy is more rapid at higher flight times. This indicates that as the $10 \mu$ s window moves to shorter flight times some contribution of the other processes 1 and 2 is present.

The excitation functions in Figs. 8(b) and 8(c) show contributions due to multiple processes. Careful examination of the excitation functions for the individual $10 \mu$ s windows does not reveal any new onsets besides the ones identified above.

An equivalent set of excitation functions has been measured with the detector at $45^{\circ}$. The excitation functions at $45^{\circ}$ are almost identical in shape to the excitation functions at $90^{\circ}$ and the onsets have been measured at 11.7, 13.5, and $30.3 \mathrm{eV}$. Within experimental uncertainty these values agree with the values found at $90^{\circ}$. This uncertainty is estimated to be $1.0 \mathrm{eV}$ and depends both on the energy resolution of the incident electron beam (about $0.8 \mathrm{eV}$ FWHM) and the statistics of the accumulated counts in the excitation function. In the remainder of this paper we have adopted the average values 11.6, 13.6, and $30.8 \mathrm{eV}$. The excitation functions measured at $45^{\circ}$ for time-of-flight windows between 220 and 520 $\mu$ s are dominated by processes 3 and 4 . They are very similar in shape as Fig. 8(d) and the observed onset is at the same position. This suggests that there is a similarity between process 3 and process 4 , even though the velocities of the fragments produced are different.

In the study of molecular dissociation the location of onsets as a function of flight time is a valuable source of information. In the case of a dissociation producing two fragments, a plot of the onset energies (appearance potentials) as a function of the kinetic energies of the fragments (for the corresponding time-of-flight intervals) would result in a straight line with a slope given by the ratio of the mass of the undetected fragment to the mass of the molecule before dissociation. It is not possible to make such a plot in the present instance, because the vast majority of fragments possess energies less than $1 \mathrm{eV}$ and the uncertainty in the onsets is of similar order.

\section{Kinetic energy distributions}

The procedure described in Sec. III C has allowed us to obtain a series of time-of-flight spectra at $90^{\circ}$, containing contributions of processes 1 and 2 only. Kinetic energy distributions for these processes have been obtained by using the $t_{M} \rightarrow t$ transformation and the $t \rightarrow E$ transformation in succession. We have used $v=600 \mathrm{~m} / \mathrm{s}, l=124 \mathrm{~mm}$, and $m=40 \mathrm{amu}$ in the transformations, assuming that single argon atoms are detected. Figure 9 shows the time-of-flight spectra obtained after subtraction of the fits and application of the $t_{M} \rightarrow t$ transformation. Figure 10 shows the kinetic energy distributions obtained after application of the $t \rightarrow E$ transformation to the spectra of Fig. 9.

Whereas the $t_{M} \rightarrow t$ transformation is almost linear below $200 \mu$ s (see Fig. 4), the $t \rightarrow E$ transformation is very nonlinear and as a result one feature may dominate the kinetic energy distribution. This is found to be the case when the transformations are applied to the time-of-flight spectra at $45^{\circ}$. The kinetic energy distributions are dominated by a large peak below $0.2 \mathrm{eV}$ with a maximum at $0.05 \mathrm{eV}$, which obscures the contribution due to the processes 1 and 2 . The peak is mainly produced by process 3 , but may also be affected by inaccuracies in the subtraction of the fits.

We have also attempted to transform the time-of-flight spectra at $90^{\circ}$, without subtracting the fits. The kinetic energy distributions obtained are again dominated by process 3 , producing a large peak below $0.2 \mathrm{eV}$ with a maximum near 


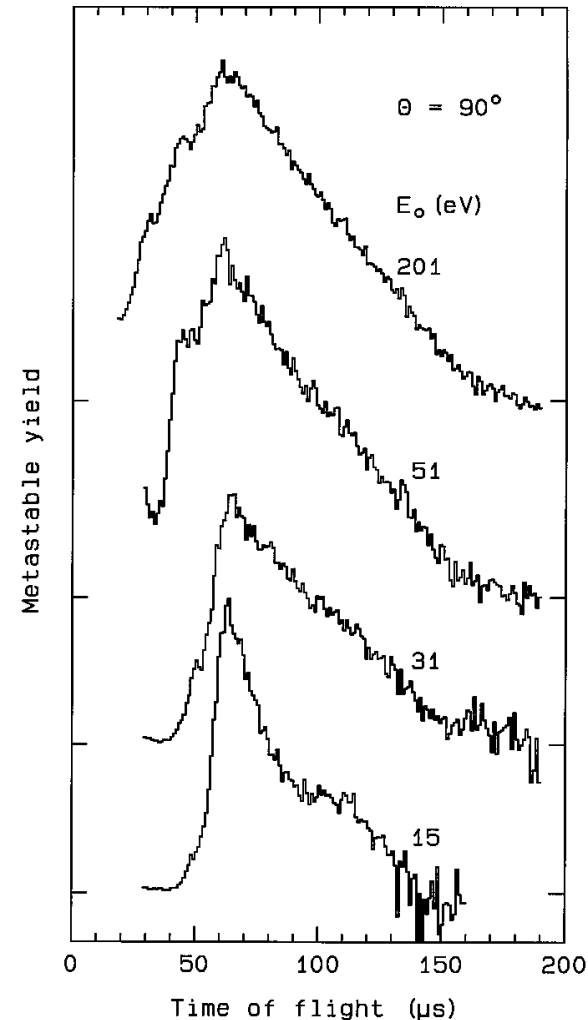

FIG. 9. Time-of-flight spectra for neutral metastable fragments produced by processes 1 and 2 (see text) and detected at $90^{\circ}$. Each spectrum has been obtained from the corresponding time-of-flight spectrum in Fig. 5(b) by subtraction of the contribution of process 3 , and application of a transformation from laboratory time-of-flight to beam time-of-flight (see text). Vertical scales are comparable except the bottom spectrum is multiplied by 4 .

$0.1 \mathrm{eV}$. However, this distribution may be affected by the small background of argon atoms present in the vacuum chamber. Because the transformation to kinetic energy is very nonlinear and therefore very sensitive to inaccuracies, the kinetic energy distributions we have obtained for process 3 are unreliable.

Estimates for the most probable kinetic energy of the fragments produced by process 4 can be obtained from the maxima in the time of flight spectra at $45^{\circ}$. The maximum occurs at $t_{M}=210 \mu \mathrm{s}$ (see Fig. 7), corresponding to $t=270$ $\mu$ s and $E=0.044 \mathrm{eV}$.

\section{DISCUSSION}

Based on the information obtained from the time-offlight spectra, the excitation functions and the kinetic energy distributions we have identified four different processes contributing to the observed spectra.

(1) A process with an onset at $13.6 \mathrm{eV}$ and a maximum at 20 $\mathrm{eV}$, producing fragments with about $0.2-1.0 \mathrm{eV}$ kinetic energy.

(2) A process with an onset at $30.8 \mathrm{eV}$ and a maximum at a much higher energy, producing fragments with about $0.2-1.0 \mathrm{eV}$ kinetic energy. A small number of the fragments have energies somewhat higher than $1 \mathrm{eV}$.

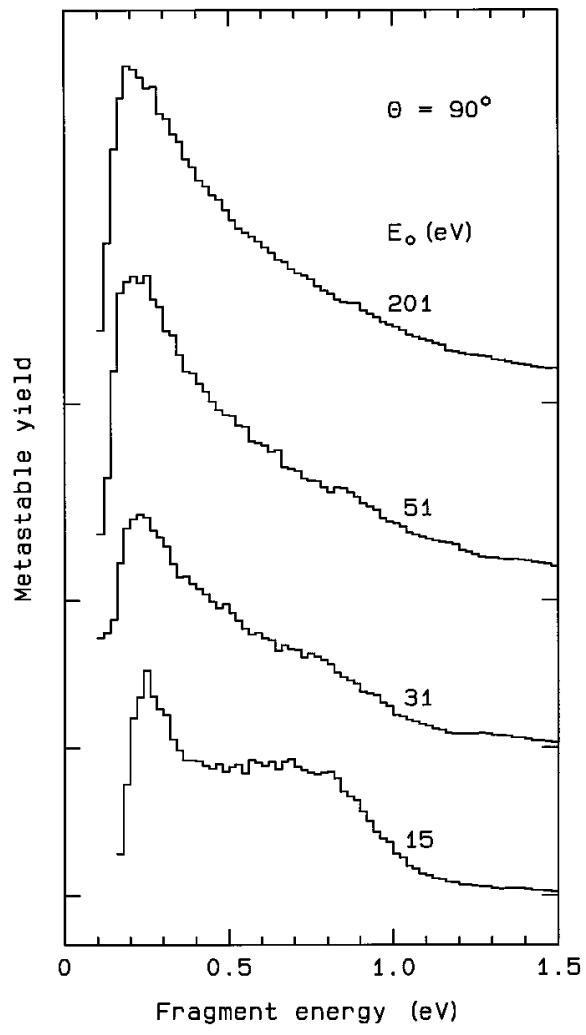

FIG. 10. Kinetic energy distributions of neutral metastable fragments produced by processes 1 and 2 (see text) and detected at $90^{\circ}$. Each spectrum has been obtained from the corresponding spectrum in Fig. 9 by application of a transformation of beam time-of-flight to fragment kinetic energy (see text). Vertical scales are comparable except the bottom spectrum is multiplied by 5 .

(3) A process with an onset at $11.6 \mathrm{eV}$, producing fragments below $0.2 \mathrm{eV}$ with a maximum at about $0.1 \mathrm{eV}$ kinetic energy.

(4) A process with an onset at $11.6 \mathrm{eV}$ and a maximum at 20 $\mathrm{eV}$. These are the slowest fragments and they are only detected at $45^{\circ}$. Their average kinetic energy is about $0.04 \mathrm{eV}$.

Regarding the interpretation of these processes two related fields of research are of relevance. These fields are the ionization of noble-gas clusters, and the formation of excitons in noble-gas solids and clusters. The model now generally accepted for the ionization of noble-gas clusters (Haberland ${ }^{42}$ ) is the following. After the cluster has been ionized by electron or photon impact, the cluster is left with a positively charged hole which becomes localized (on a ps time scale) in a molecular ion within the cluster. The vibrational relaxation of this ion releases about $1 \mathrm{eV}$ of energy into the cluster, causing the evaporation of several groundstate atoms from the cluster, before the ion is detected in a mass spectrometer. While the initial model evoked a dimer ion core, ${ }^{42}$ more recent experimental ${ }^{20,37,38,43-45}$ and theoretical ${ }^{46}$ research has indicated that in $\mathrm{Ar}_{n}^{+}$cluster ions with $n<15$ the positive charge is localized in a trimer ion core.

The physics of excitons in noble-gas solids is a broad field of research, ${ }^{53-55}$ while extensive research on excitons in 
noble-gas clusters has been performed by Möller and co-workers. ${ }^{7,8,19,21-23}$ Using fluorescence excitation spectroscopy, they observed the absorption bands of the noble-gas clusters to shift towards the energies of the excitons in the solid noble gases with increasing average cluster size. Based on these results we propose that the process 1 proceeds through the following sequence of steps:

(a) Excitation of an exciton state in the cluster. The incident electron excites the cluster and leaves without further interaction. Wörmer et al. ${ }^{8}$ show that in smaller argon clusters the lowest $n=2$ excitons occur at 13.1 and $13.7 \mathrm{eV}$. These values are in very good agreement with the onset of process 1 we observe at $13.6 \mathrm{eV}$. The experimental uncertainty of our onset is about $1 \mathrm{eV}$, and is insufficient to determine which of the $n=2$ exciton levels we observe.

(b) Localization of the exciton in an excimer or excited trimer located within the cluster. The time scale for the localization is of the order of a few ps. ${ }^{28}$ The size of the localized exciton and also its location within the cluster is open to debate. $\mathrm{Ar}_{2}$ potentials leading to $\operatorname{Ar}^{*}\left(3 p^{5} 4 p\right)$ are very similar ${ }^{52}$ to the $\mathrm{Ar}_{2}^{+}$ground state potential, suggesting that similar to $\mathrm{Ar}_{n}^{+}$clusters, the localized exciton could well be a trimer in an excited state. However, luminescence spectra of excitons in noble-gas solids are generally interpreted in terms of localizations occurring on monomer and excimer sites in the crystal, ${ }^{27,53-55}$ and no mention is made of trimers. Last and George ${ }^{52}$ have performed the only theoretical study of Rydberg excited $\mathrm{Ar}_{n}^{*}$ clusters, but their results for the higher excited states are limited because of lack of knowledge of the $\mathrm{Ar}_{2}^{*}$ potentials. ${ }^{29}$ However, they do report relatively tightly bound states of the trimer based on $\mathrm{Ar}^{*}{ }^{1} D(4 p)$ and suggest that more highly excited $\mathrm{Ar}$ atoms should be well capable of forming metastable clusters similar to those where ionization is involved. Further theoretical studies would be helpful in clarifying this issue.

(c) Vibrational relaxation of the excimer or excited trimer. Landman et al. ${ }^{34}$ and Scharf et al. ${ }^{35}$ have studied the excited-state dynamics of excimers in $\mathrm{Ar}_{13}^{*}$ and $\mathrm{Xe}_{2}^{*} \mathrm{Ar}_{11}$ clusters. Their numerical simulations show that energy exchange between the excimer and the cluster results in the evaporation of several single ground-state atoms with very low kinetic energies (about $0.03 \mathrm{eV} \max$ ) over a time span of the order of 200 ps. Ground-state atoms with such low kinetic energies cannot be detected in our experiment.

(d) Fragmentation of the excimer or excited trimer. The fragmentation is only observed in our experiment provided that the localized exciton decays to a repulsive potential curve resulting in the ejection of an $\mathrm{Ar}^{*}\left(3 p^{5} 4 s\right)$ metastable atom with sufficient kinetic energy to reach the detector $(>0.037 \mathrm{eV})$, or of another excited atom that can decay radiatively to $\mathrm{Ar}^{*}\left(3 p^{5} 4 s\right)$. If we assume that the localized exciton is an excimer, we can obtain an estimate for the energy of the metastable fragment based on the $\mathrm{Ar}_{2}$ potential curve dia-

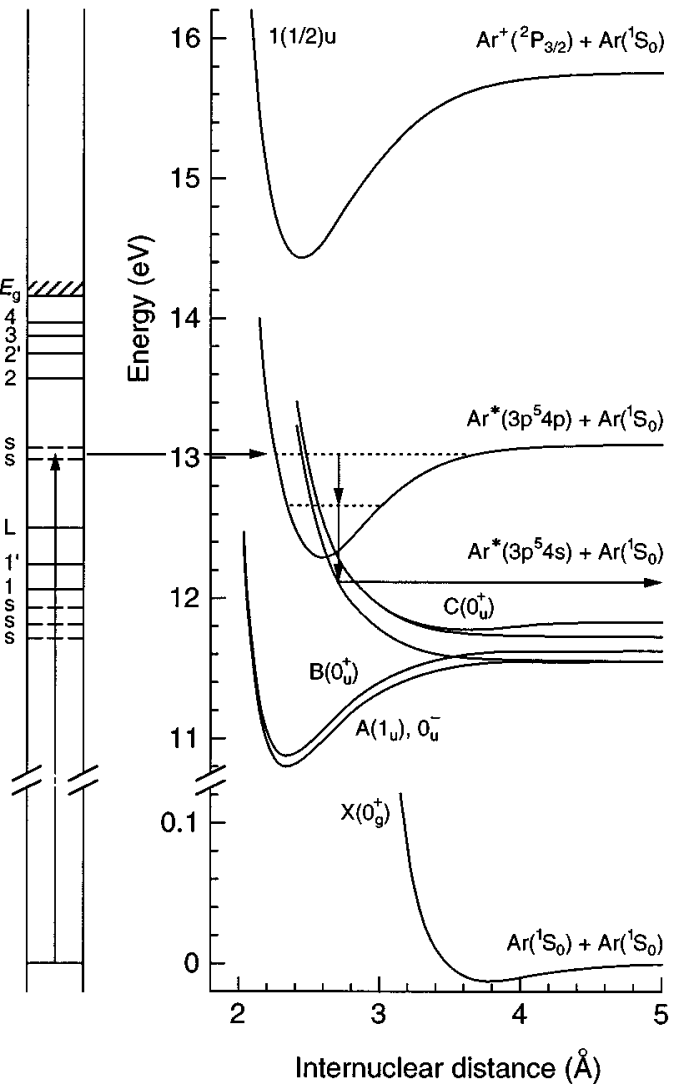

FIG. 11. (Left) Location of excitons in solid argon [Saile (Ref. 24)]. Numbers indicate principle quantum numbers of bulk excitons; $L$ is a longitudinal excitation; $S$ is a surface exciton; $E_{g}$ is the energy of the band gap. (Right) Schematic diagram of $\mathrm{Ar}_{2}$ potential curves [Mizukami and Nakatsuji (Ref. 29)]. Only two of the repulsive potential curves leading to $\operatorname{Ar*}\left(3 p^{5} 4 s\right)+\operatorname{Ar}\left({ }^{1} S_{0}\right)$ are shown. Arrows indicate a possible sequence of steps occurring in electron-impact fragmentation of argon clusters (assuming the localized exciton is an excimer; see discussion of process 1 in the text).

gram. Figure 11 shows a few potential energy curves for $\mathrm{Ar}_{2} \cdot{ }^{29-33}$ After localization the excimer is in an excited state of one of the bound potential curves associated with $\operatorname{Ar}^{*}\left(3 p^{5} 4 p\right)$ with energies between $12.91 \mathrm{eV}$ and $13.48 \mathrm{eV}$. A metastable atom is ejected if the excimer makes a transition to one of the repulsive potential curves leading to $\operatorname{Ar} *\left(3 p^{5} 4 s\right)+\operatorname{Ar}\left({ }^{1} S_{0}\right)$ with asymptotic energies of 11.55 and $11.72 \mathrm{eV}$. Based on the calculated curves, the energy of the detected fragments is expected to range from a few tenths of an $\mathrm{eV}$ up to 1 $\mathrm{eV}$, in good agreement with the energies observed in Fig. 10.

So far we have assumed that the detected fragments are metastable argon atoms. Last and George ${ }^{52}$ comment on the possible existence of metastable $\mathrm{Ar}_{2}^{*}$ and $\mathrm{Ar}_{3}^{*}$. If such fragments would be produced, they would only be detected provided that they acquire enough kinetic energy during fragmentation of the cluster. It seems that only through dissociation is enough energy available, and it is unlikely that larger fragments are observed in our experiment.

There are other possibilities in the last step of the process described, that do not lead to the ejection of a metastable atom. The dissociation of the localized exciton may 
lead to the ejection of an argon atom in an excited state which decays by emission of an ultraviolet photon. Alternatively the localized exciton may decay to a ground state configuration by emission of an ultraviolet photon. Verkhovtseva et $a l .{ }^{36}$ have studied these ultraviolet emissions for argon and krypton clusters as a function of average cluster size. These measurements are very interesting in relation to our results. However, Verkhovtseva et al. ${ }^{36}$ have used an electron impact energy of $1 \mathrm{keV}$. It would be very interesting to repeat these measurements at low electron energies.

Process 2 produces fragments with kinetic energies in the same range as process 1 . The onset for process 2 is at $30.8 \mathrm{eV}$, which is (unlike the onset for the other processes) above the ionization threshold of the cluster. There are in principle three possible explanations for this onset. Double excitation of the clusters is one possibility, however, the excitation of two $n=2$ excitons in the clusters would have a threshold near $27 \mathrm{eV}$, somewhat below the observed onset. Simultaneous excitation and ionization has a threshold near $28 \mathrm{eV}$, which is the sum of the excitation energy of an $n=2$ exciton and the ionization potential of the clusters $(14.3 \mathrm{eV}$, see Ganteför et al. ${ }^{27}$ and Kamke et $a l .^{38}$ ). Double ionization has a threshold near $32 \mathrm{eV}$ (Scheier and Mark ${ }^{39}$ ).

Support for the simultaneous excitation and ionization comes from the measurements of Foltin et al. ${ }^{40}$ and Steger et $a .^{41}$ Foltin et $a l .{ }^{41}$ have observed a process with an onset at $27 \mathrm{eV}$ in the metastable decay of $\mathrm{Ar}_{n}^{*}$ ions leading to the loss of about 3-6 monomers, which they attribute to the simultaneous excitation and ionization of the neutral clusters. Steger et ll $^{41}$ have observed the same onset in the metastable decay of $\mathrm{Ar}_{4}^{+}$to $\mathrm{Ar}_{2}^{+}$. It is possible that we observe the same process, assuming that the $n=2$ exciton decays in a very similar way as described for process 1 .

Buchenau et al. ${ }^{5}$ have measured excitation functions for the production of $\mathrm{He}^{+}$and $\mathrm{He}_{2}^{+}$by electron impact on helium clusters. These excitation functions show two onsets at $21 \mathrm{eV}$ and $40 \mathrm{eV}$, the latter onset being attributed to the double excitation of the helium clusters. The qualitative similarity between the $\mathrm{He}^{+}$excitation function and our excitation function in Fig. 8(a) is striking. In view of the large number of possible excited states in the clusters it is surprising that only two onsets are observed in these excitation functions.

The lower onset of $11.6 \mathrm{eV}$ for the slower fragments produced by processes 3 and 4 indicates that the excitation of the cluster proceeds via an $n=1$ exciton. These excitons have been observed between 11.6 and 12.2 eV. ${ }^{6,8,21}$ The process leading to the ejection of a metastable atom may be very similar to the electron and photon stimulated desorption of noble-gas solids. ${ }^{26,56-58}$ This process involves the localization of an exciton onto a single excited atom causing a deformation of the lattice due to the weakly repulsive interaction with neighboring atoms. A cavity is formed around the localized exciton, causing its ejection. Kinetic energies of the desorped atoms are of the order of $0.1 \mathrm{eV}^{26,57,58}$

Last and George ${ }^{52}$ have done extensive numerical calculations of small clusters in electronic states associated with $\operatorname{Ar}^{*}\left(3 p^{5} 4 s\right)$. They argue that the exciton tends to locate at the periphery of the cluster. $\mathrm{The}_{2}^{*}$ potentials asymptotic to $\operatorname{Ar}^{*}\left(3 p^{5} 4 s\right)$ are weakly repulsive at large interatomic dis- tances, causing the formation of the cavity around the localized exciton. The fact that two processes are observed in our measurements may perhaps point to the existence of localized excitons as either monomers or excimers. The $\mathrm{C}\left(O_{u}^{+}\right)$ potential has a shallow well, supporting an excimer state, which could dissociate to $\operatorname{Ar} *\left(3 p^{5} 4 s\right)+\operatorname{Ar}\left({ }^{1} S_{0}\right)$, producing a metastable atom with about $0.05 \mathrm{eV}$ kinetic energy.

Whereas the present experiment looks at fragments from neutral argon clusters, there are several studies of fragments from ionized argon clusters. These studies involve kinetic energy distributions of both ionized and neutral fragments. The advantage of studying ionized clusters is that a beam of only one cluster size can be produced by ionization of a neutral cluster beam followed by time-of-flight mass analysis.

Stace and co-workers $9,47,48$ and Nagata and co-workers ${ }^{10,49,50}$ have measured kinetic energy distributions of both ionized and neutral fragments produced by photodissociation of argon cluster ions. In both experiments a fast beam of mass-resolved $\mathrm{Ar}_{n}^{+}$cluster ions is crossed with a 532 $\mathrm{nm}$ laser beam and the photofragments are detected using a time-of-flight technique. By analysis of the photofragment time-of-flight signals both groups have obtained information on the kinetic energy distributions of the fragments. Two types of neutral fragments have been observed. Slow fragments with energies of $0-0.1 \mathrm{eV}$ are attributed to the evaporation of atoms. Fast fragments with energies of $0.1-0.6 \mathrm{eV}$ are attributed to dissociation of the chromophoric $\mathrm{Ar}_{3}^{+}$core in the cluster.

The similarities in the kinetic energy distribution of neutral fragments from cluster ions (Smith et al., ${ }^{9}$ Nagata and Kondow $^{10}$ ) and of neutral fragments from neutral clusters (present experiment) originate from the analogy between the underlying mechanisms. In the experiments of Smith et al. ${ }^{9}$ and of Nagata and Kondow ${ }^{10}$ the fast fragments are produced by dissociation of the central $\left(\mathrm{Ar}_{3}^{+}\right)^{*}$ core of the cluster; in our experiment the fragments are produced by dissociation of a excimer or excited trimer within the cluster.

\section{CONCLUSION}

We have presented time-of-flight measurements of neutral metastable fragments produced by electron collisions with neutral argon clusters. Four different production mechanisms for neutral metastable fragments have been identified and kinetic energy distributions for the faster fragments have been obtained. We have interpreted the fragmentation of the clusters in terms of excitation and decay of excitons in the clusters. The faster fragments are produced by $n=2$ excitons, which localize on an excimer or an excited trimer within the cluster and upon dissociation cause the ejection of a metastable atom. The slower fragments are produced by $n=1$ excitons, which tend to localize on the periphery of the cluster, leading to the ejection of a metastable atom due to weak repulsive forces with neighboring atoms.

The present results indicate possible fruitful areas of future research. Measurements of yields of metastable atoms, and also yields of photoemissions for relevant transitions should provide additional information about the fragmenta- 
tion processes observed. These yields would be relevant both as a function of electron impact energy and as a function of average cluster size.

\section{ACKNOWLEDGMENTS}

The authors are happy to acknowledge financial assistance from the Natural Sciences and Engineering Research Council of Canada and expert technical help from the mechanical and electronic workshop staff at the University of Windsor. Helpful discussions with Dr. T. F. George are gratefully acknowledged. We are especially indebted to Mr. W. Grewe for his essential contributions to the mechanical design of the apparatus and to Lisa Pastorius for help with some of the measurements.

${ }^{1}$ U. Buck and H. Meyer, J. Chem. Phys. 84, 4854 (1986).

${ }^{2}$ T. D. Märk, P. Scheirer, K. Leiter, W. Ritter, K. Stephan, and A. Stamatovic, Int. J. Mass. Spectrom. Ion. Phys. 74, 281 (1986).

${ }^{3}$ T. D. Märk, Z. Phys. D 12, 263 (1989).

${ }^{4}$ J. Gspann and H. Vollmar, J. Chem. Phys. 73, 1657 (1980).

${ }^{5}$ H. Buchenau, J. P. Toennies, and J. A. Northby, J. Chem. Phys. 95, 8134 (1991).

${ }^{6}$ A. Burose, C. Becker, and A. Ding, Z. Phys. D. 20, 35 (1991).

${ }^{7}$ J. Stapelfeldt, J. Wörmer, and T. Möller, Phys.Rev. Lett. 62, 98 (1989).

${ }^{8}$ J. Wörmer, M. Joppien, G. Zimmerer, and T. Möller, Phys. Rev. Lett. 67, 2053 (1991).

${ }^{9}$ J. A. Smith, N. G. Gotts, J. F. Winkel, R. Hallett, C. A. Woodward, A. J. Stace, and B. J. Whitaker, J. Chem. Phys. 97, 397 (1992).

${ }^{10}$ T. Nagata and T. Kondow, J.Chem. Phys. 98, 290 (1993).

${ }^{11}$ R. S. Freund, in Rydberg States of Atoms and Molecules, edited by R. F. Stebbings and F. B. Dunning (Cambridge University, Cambridge, 1983), p. 355 .

${ }^{12}$ R. N. Compton and N. Bardsley, in Electron-Molecule Collisions, edited by I. Shimamura and K. Takayanagi (Plenum, New York, 1984), p. 275.

${ }^{13}$ E. C. Zipf, in Electron-Molecule Interactions and their Applications, edited by L. G. Christophorou (Academic, New York, 1984), Vol. 1.

${ }^{14}$ W. C. Wiley and I. H. McLaren, Rev. Sci. Instrum. 26, 1150 (1955).

${ }^{15}$ P. J. M. van der Burgt and J. W. McConkey, J. Phys. B 24, 4821 (1991).

${ }^{16}$ P. J. M. van der Burgt, M. E. Antaya, and J. W. McConkey, Z. Phys. D 24, 125 (1992).

${ }^{17}$ L. LeClair, M. Sc. thesis, University of Western Ontario, London, Ontario, 1989.

${ }^{18}$ O. F. Hagena, Z. Phys. D. 4, 291 (1987).

${ }^{19}$ J. Wörmer, V. Guzielski, J. Stapelfeldt, G. Zimmerer, and T. Möller, Phys. Scr. 41, 490 (1990).

${ }^{20}$ N. E. Levinger, D. Ray, M. L. Alexander, and W. C. Lineberger, J. Chem. Phys. 89, 5654 (1988)

${ }^{21}$ J. Wörmer and T. Möller, Z. Phys. D 20, 39 (1991).

${ }^{22}$ T. Möller, Z. Phys. D 20, 1 (1991).

${ }^{23}$ T. Möller and G. Zimmerer, J. Opt. Soc. Am. B 6, 1062 (1989).
${ }^{24}$ V. Saile, Appl. Opt. 19, 4115 (1980).

${ }^{25}$ S. Bernstorff and V. Saile, Opt. Commun. 58, 181 (1986).

${ }^{26}$ T. Kloiber and G. Zimmerer, Phys. Scr. 41, 962 (1990).

${ }^{27}$ J. Jortner, L. Meyer, S. A. Rice, and E. G. Wilson, J. Chem. Phys. 42, 4250 (1965).

${ }^{28}$ E. S. Peterson, B. J. Schwartz, and C. B. Harris, J.Chem. Phys. 99, 1693 (1993).

${ }^{29}$ Y. Mizukami and H. Nakatsuji, J. Chem. Phys. 92, 6084 (1990).

${ }^{30}$ D. C. Shannon and J. G. Eden, J. Chem. Phys. 89, 6644 (1988).

${ }^{31}$ P. R. Herman, P. E. LaRocque, and B. P. Stoicheff, J. Chem. Phys. 89, 4535 (1988).

${ }^{32}$ M. C. Castex, M. Morlais, F. Spiegelmann, and J. P. Malrieu, J. Chem. Phys. 75, 5006 (1981).

${ }^{33}$ W. R. Wadt, J. Chem. Phys. 68, 402 (1978).

${ }^{34}$ U. Landman, R. N. Barnett, C. L. Cleveland, D. Scharf, and J. Jortner, J. Phys. Chem. 91, 4890 (1987).

${ }^{35}$ D. Scharf, J. Jortner, and U. Landman, J. Chem. Phys. 88, 4273 (1988).

${ }^{36}$ E. T. Verkhovtseva, E. A. Bondarenko, and Yu. S. Doronin, Chem. Phys. Lett. 140, 181 (1987).

${ }^{37}$ G. Ganteför, G. Bröker, E. Holub-Krappe, and A. Ding, J. Chem. Phys. 91, 7972 (1989).

${ }^{38}$ W. Kamke, J. de Vries, J. Krauss, E. Kaiser, B. Kamke, and I. V. Hertel, Z. Phys. D 14, 339 (1989).

${ }^{39}$ P. Scheier and T. D. Mark, Chem. Phys. Lett. 136, 423 (1987).

${ }^{40}$ M. Foltin, G. Walder, A. W. Castleman, Jr., and T. D. Mark, J. Chem. Phys. 94, 810 (1991).

${ }^{41}$ H. Steger, J. de Vries, W. Kamke, and I. V. Hertel, Z. Phys. D 21, 85 (1991).

${ }^{42}$ H. Haberland, Surf. Sci. 156, 305 (1985).

${ }^{43}$ K. Norwood, J.-H. Guo, and C. Y. Ng, J. Chem. Phys. 90, 2995 (1989).

${ }^{44}$ H. Haberland, T. Kolar, C. Ludewigt, A. Risch, and M. Schmidt, Z. Phys. D 20, 33 (1991a).

${ }^{45} \mathrm{H}$. Haberland, B. von Issendorff, T. Kolar, H. Kornmeier, C. Ludewigt, and A. Risch, Phys. Rev. Lett. 67, 3290 (1991b).

${ }^{46}$ T. Ikegami, T. Kondow, and S. Iwata, J. Chem. Phys. 98, 3038 (1993).

${ }^{47}$ C. A. Woodward and A. J. Stace, J. Chem. Phys. 94, 4234 (1991).

${ }^{48}$ J. A. Smith, J. F. Winkel, A. B. Jones, A. J. Stace, and B. J. Whitaker, J. Chem. Phys. 100, 6412 (1994); G. H. Dunn, Phys. Rev. Lett. 8, 62 (1962).

${ }^{49}$ T. Nagata, J. Hirokawa, and T. Kondow, Chem. Phys. Lett 176, 526 (1991).

${ }^{50}$ T. Nagata, J. Hirokawa, T. Ikegami, T. Kondow, and S. Iwata, Chem. Phys. Lett. 171, 433 (1990).

${ }^{51}$ H. Haberland, U. Buck, and M. Tolle, Rev. Sci. Instrum. 56, 1712 (1985).

${ }^{52}$ I. Last and T. F. George, J. Chem. Phys. 98, 6406 (1993).

${ }^{53}$ I. Ya. Fugol', Adv. Phys. 27, 1 (1978).

${ }^{54}$ I. Ya. Fugol', Adv. Phys. 37, 1 (1988).

${ }^{55}$ N. Schwentner, E.-E. Koch, and J. Jortner, Electronic Excitations in Condensed Rare Gases, Springer Tracts in Modern Physics (Springer, Berlin, 1985), Vol. 107.

${ }^{56}$ F. Coletti, J. M. Debever, and G. Zimmerer, J. Phys. Lett. 45, L-467 (1984).

${ }^{57}$ D. J. O’Shaughnessy, J. W. Boring, S. Cui, and R. E. Johnson, Phys. Rev. Lett. 61, 1635 (1988).

${ }^{58}$ S. Cui, R. E. Johnson, and P. T. Cummings, Phys. Rev. B 39, 9580 (1989). 